Manuscript received July 23 , 2020; accepted for publication September 22, 2020; published online January 7, 2021. Issue published September 1, 2021.

1 Department of Architecture, Bilkent University, Üniversiteler, 1608. St. No: 50, Ankara 06800 , Turkey, (i) http://orcid.org/00000001-5619-1975

2 Department of Architecture, Bilkent University, Üniversiteler, 1608. St. No: 50, Ankara 06800, Turkey (Corresponding author), e-mail: zuhre@bilkent.edu.tr, (1) http://orcid.org/0000-00023655-9282
Beyza Yazıcl ${ }^{1}$ and Zühre Sü Gül ${ }^{2}$

\section{Biomimetic Metamaterial Design Simulation and Evaluation for Building Acoustics by Impedance Measurements}

\section{Reference}

B. Yazıcı and Z. S. Gül, "Biomimetic Metamaterial Design Simulation and Evaluation for Building Acoustics by Impedance Measurements," Journal of Testing and Evaluation 49, no. 5 (September/ October 2021): 3183-3203. https://doi.org/10.1520/JTE20200469

\section{ABSTRACT}

This study investigates the potentials of acoustic metamaterial (AMM) applications in room and building acoustics by means of impedance tube experiments. With their extreme properties in either sound absorption or transmission loss, AMMs can perform better than traditional acoustic materials in buildings. Importantly, they are also more sustainable and hygienic than fibrous and porous materials. Depending on the matrix material used, AMMs can vary in transparency and color. Considering both their acoustic and aesthetic values, this study develops different types of metamaterial for possible uses as a partition wall, a surface layer, or a design element. The proposed metamaterials are primarily based on the exploration of ratios and forms from nature-the golden ratio, web-labyrinthine structures, genetic and neural systems such as deoxyribonucleic acid (DNA) molecules, and the synapse structures in the brain-reproduced on a subwavelength scale. These abstractions are then combined with the 3-D space coiling and 3-D labyrinth approaches of AMM design. Modules of the proposed AMMs are manufactured in a 3-D printer and tested in an impedance tube to estimate their normal incidence sound absorption coefficients and transmission loss characteristics. Based on the results obtained, the modules with the higher performances are used in the design of partition walls of varying heights. Two real-case architecture studios are simulated with and without the proposed AMM interventions over field test-tuned acoustical models of the studios to assess the effectiveness of such an approach in a possible acoustical design problem.

\section{Keywords}

acoustic metamaterial, sound absorption, transmission loss, impedance tube measurements, building acoustics 


\section{Introduction}

Research on materials is a fundamental topic in room and building acoustics. A range of materials are either applied in construction systems for noise control or used as a surface finish layer or decorative object for reverberation control. Advances in technology have made it possible to control sound within the structure of novel materials. ${ }^{1}$ Meanwhile, green technology considerations have brought in new requirements for buildings such as hygiene standards, limitations on toxic gas emissions, and recyclability or reusability. One of the objectives of this research is to combine the form, structure, and technology of materials to develop sustainable, ecofriendly acoustical design solutions for use in room and building acoustics.

Trends in materials change with advancing technology. Metamaterials are engineered materials consisting of small metastructures with nontraditional effective properties. ${ }^{2}$ Studies on artificial structures commenced with phononic $^{3}$ crystals analogous to the photonic ${ }^{4}$ crystals used in optics. There are various fields of research on metamaterials as of the pantographic structures. The design procedure utilizes mathematical models and numerical simulations, which are then supported by experiments. ${ }^{5,6}$ Recently, research into acoustical materials has acquired new perspectives as a result of the introduction of metamaterial applications in acoustic cloaking, ${ }^{7-10}$ imaging, ${ }^{11}$ focusing and lensing ${ }^{12}$ via negative refraction, ${ }^{13-15}$ and surface wave manipulation. ${ }^{16}$

The use of the term "meta" stems from the unusual properties of designed acoustic metamaterials (AMMs). Physical properties like negative mass density, negative bulk modulus, or negative effective refractive index values ${ }^{17}$ result in extreme acoustic performance. Many AMMs have been proposed to adjust the effective quantity to these extreme values, including material properties, like negative ${ }^{18}$ and near-zero values, ${ }^{19}$ or to obtain high anisotropic density. ${ }^{20}$ Locally resonant AMMs are of special interest because of their subwavelength scale compared with their unit cell sizes. In one of the early studies on artificial AMMs, Liu et al. ${ }^{21}$ used rubber-coated spheres for subwavelength performance, utilizing a locally resonant construction in a periodic structure to manipulate acoustic waves, although Liu et al.'s early work is highly limited by the functioning frequency range. Negative density can be stimulated by the local resonance of the structure. Inspired by results obtained in electromagnetics, Fang et al. ${ }^{22}$ introduced a new type of AMM, including a sequence of Helmholtz resonators. In this way, they achieved a negative bulk modulus value near the bandgap. Other new AMMs that have emerged include perforated plate-type AMMs, ${ }^{7,23}$ space-coiling-type AMMs, ${ }^{12,13,15,16,24-26}$ and membrane-and-plate-type AMMs. ${ }^{18}$

One of the first studies on membrane-type AMMs is by Yang et al., ${ }^{27}$ who proposed manipulating the sound waves in the low-frequency range between 50 and 1,000 $\mathrm{Hz}$ by ensuring negative mass density in the bandgap. A broadband sound shield is investigated to incorporate simple stacking of membrane-type metamaterials operating at different frequency regimes. Some advantages of membrane-and-plate-type AMMs are that they are generally lightweight structures and thus desirable materials for the aerospace and automotive sectors, wherein performance and weight are both important. Their geometrical forms are less complex than those of other types of $\mathrm{AMM}$, and their effective density can be optimized easily. ${ }^{28}$

Another group of AMMs embodies the labyrinthine or space-coiling approach, which helped to stimulate this study. With coiled-up spaces, it is possible to create efficient geometrical configurations. Research has shown that AMMs of this type offer several benefits, such as including a remarkable degree of sound absorption because of the isotropic response related to their highly symmetrical nature ${ }^{26}$ One of the very first studies on space-coiled AMMs is by Liang and Li. ${ }^{15}$ By applying curled perforations, they extended the transmission pathway that sound waves follow inside the material to make it much longer than the external dimensions. As a result, their proposed metamaterial displayed double negativity and a large refractive index. Xie et al. ${ }^{16}$ demonstrated by experiment that the AMM of a suitably designed labyrinth could achieve a wide frequency range of the negative refractive index. They experimented with quasi-2-D labyrinthine AMMs. Frenzel et al. ${ }^{29}$ made possible the transition from 2-D labyrinth-type metamaterials to 3-D metamaterials with the objective of devising a broadband all-angle acoustic absorber.

In general, fabricated acoustic metasurfaces exhibit extreme sound absorption with multipolar properties at the monopolar resonant frequency. Most importantly, space-coiling AMMs can achieve adjustable operating 
frequency by varying the number of curls for higher reflection. ${ }^{26}$ Other metamaterials incorporating space coiling in their 3-D structures utilize a zigzag movement to increase the efficient sound path, as the zigzag movement leads to wave-front manipulation ${ }^{30}$ and extreme acoustic transmission. ${ }^{15,29}$ In addition to the zigzag path, the clockwise-anticlockwise direction has been shown to be effective in reducing the energy of sound waves. Liu, Xia, and $\mathrm{Yu}^{31}$ proposed a spiral and labyrinthine AMM, with transmission path in this form, which is considerably different from the zigzag path. They were able to cut off the sound waves in the waveguide effectively by forcing them to propagate alternatively through the path in a clockwise direction and an anticlockwise direction. A study done by Godbold, Soar, and Buswell ${ }^{32}$ proposed a novel sound absorber based on the principle of passive destructive interference (PDI), addressing its technical performance, manufacture, and design for room acoustics applications. Their findings showed that the original absorber they proposed might constitute a desirable alternative to the conventional Helmholtz resonator since the Helmholtz device requires a bulky cavity to operate, while the novel absorber needs less space. In another study making use of PDI, Setaki et al. ${ }^{33}$ compared experimental and analytical estimates of a material containing cylindrical structures of different lengths. They investigated the sound absorption by systematically changing the inner geometry of the tubes with a view to developing an effective acoustic element for use in architectural spaces.

AMMs have attracted the attention of researchers in various fields, and new designs and classes of material are being introduced continuously. In the field of architectural acoustics, however, AMM research is still very new, and much more experimental and theoretical investigation is needed for possible real-case applications. This study is motivated by the idea of employing novel design tools for the development of AMMs with a specific focus on sound improvement and noise control in architectural spaces. Architectural applications pose challenges since parameters such as aesthetic appeal, design flexibility, fire resistance, and hygiene need to be satisfied in addition to acoustic performance requirements. The study seeks to optimize the performances of AMMs of the space-coiled type in terms of sound absorption and transmission loss (TL) at low frequencies, which is a problematic range in room acoustics, mainly by adjusting the geometry of the inner structure of the proposed AMMs. A semitransparent base material resin is selected for manufacturing, exposing the inner design and offering potential for light and color integration.

Although AMMs are named after their "beyond nature" characteristics or proportions, the limits of nature are not fully known, and there is considerable potential for abstracting forms and ratios from nature and making use of them. Another major source of inspiration for this research, therefore, is biomimicry. There are many studies on biomimicry in material science and architecture. For example, spider web geometry ${ }^{17}$ provides a strong fiber net, even stronger than steel, and has a lightweight structure. For this reason, it is frequently preferred in applications wherein both strength and light weight are critical, such as bulletproof vests or aerospace structures. ${ }^{34}$ The golden ratio (GR), which is found in nature in flowers, various fruits, vegetables, plants and ferns, spiral leaves, shells, fish, body parts of ants and human beings, the physical universe ${ }^{35}$ and so on, has caught the attention of many scientists, architects, and industrial designers in recent decades, especially in the form of the golden rectangle. Lastly, honeycomb structures, which are frequently used in metamaterials, ${ }^{36}$ are also a product of biomimicry. They are both strong and light in weight and have an optimal packing shape with their hexagonal cellular units. For these reasons, honeycomb structures are widely used in aircraft control surfaces. ${ }^{34}$

The main use of biomimicry in this study is to design features that may not be found in nature exactly but are still inspired by and abstracted from existing structures. Ratios and forms from nature are utilized on a subwavelength scale, similar to locally resonant metamaterials, within the 3-D space coiling and 3-D labyrinth approaches to AMM design. The design proposals have their origins in the GR, web-labyrinthine (WL) forms, genetic and neural systems such as deoxyribonucleic acid (DNA) molecules, and the synapse structures in the brain, as will be discussed in detail in the following sections. Modules of the sample materials designed are then manufactured in a 3-D printer. These manufactured AMM units are subsequently tested in an impedance tube to obtain their normal incidence sound absorption coefficients and TL characteristics in the frequency range below $1 \mathrm{kHz}$. Finally, to evaluate the effectiveness of the proposed AMM modules in a real-life acoustical design problem, two architecture studios are tested in their existing states and simulated with and without the AMM applications. 


\section{Design and Production}

In light of the potentials of biomimicry, the design ideas used in this study have been taken from the GR, WL forms, genetic and neural systems such as DNA molecules, and the synapse structures in the brain as detailed in the following sections. The abstraction of the original forms and their transformations into final outputs are presented in figures 1-3 for three different AMM design proposals. The sample units manufactured are limited in size to a diameter of $10 \mathrm{~cm}$ because of the impedance tube measurement setup.

\section{GR MODULE DESIGN}

The potential of the GR as an acoustic cavity in a Helmholtz resonator arrangement is integrated here with the space-coiling principle used in AMMs. Thus, the first module is inspired by the Fibonacci sequence, and the proportions ${ }^{35}$ are applied to a spiral cavity at its central aperture. The form of the GR is created using appropriate codes on a plugin named Grasshopper 3-D that runs within the Rhino 5 architectural software.

The GR system fundamentally consists of two identical units located side by side and attached to one another, with one opening on the opposite surfaces of each unit. Thus, a module contains two units of a single type, and these are placed in such a way that one unit is rotated $180^{\circ}$ along the $y$-axis to the other (fig. 1C). The spiral systems of the two units are connected at the center by an internal opening on their intersecting surfaces. The central aperture has the geometry of the GR. The voids switch to a spiral form in the direction of the outer shell of the structure after three cycles of the Fibonacci sequence have been completed at the aperture. The path of the inner void starts on one side of the first module, connects to the second module at the inner center, and ends on the other side of the second module (fig. $1 D$ and $1 E$ ). The GR sample is $10 \mathrm{~cm}$ in diameter and $7.2 \mathrm{~cm}$ in depth (fig. 1B). The length of the inner void is $153 \mathrm{~cm}$.

\section{WL MODULE DESIGN}

The second proposed AMM is named the WL system. The design idea has its origin in the spider's web in nature. ${ }^{34}$ The silk net woven by spiders in a 2 -D form is inherently very strong because of the tensile properties

FIG. 1 (A) Golden rectangle and golden spiral on an ear, (B) 3-D model of a GR-AMM prototype placed in a solid cylindrical module, (C) 3-D models of two units attached at their centers, (D) 3-D rendering of the exploded axonometric unit, $(E)$ inner structure of sample GR unit manufactured in the 3-D printer, and $(F)$ molded GR unit ready for measurement.

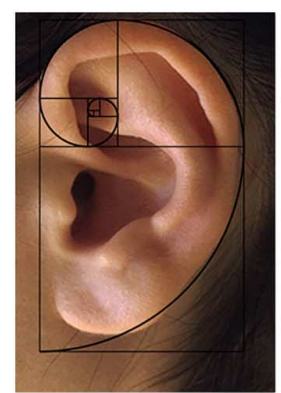

$(A)$

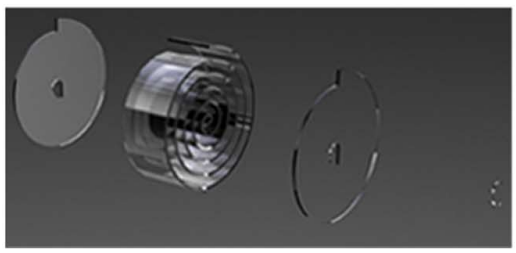

$(D)$

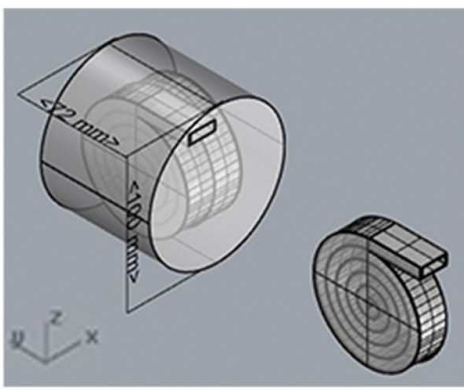

(B)

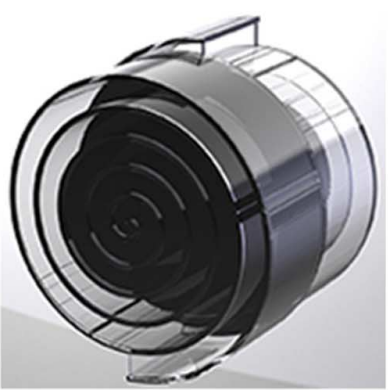

$(C)$

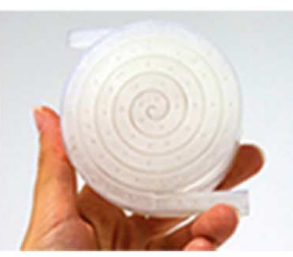

$(E)$

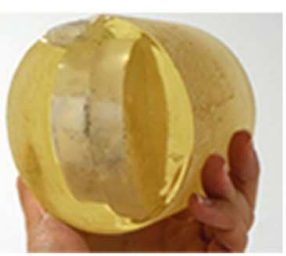

$(F)$ 
FIG. 2 (A) A spider's web as it exists in nature, (B) WL 3-D models, (C) one typical layer of the printed inner structure, $(D)$ one manufactured unit combining eight layers in actual size, $(E)$ epoxy-molded sample ready for measurement, and $(F)$ plan drawings showing the phases of the $\mathrm{WL}$ design.

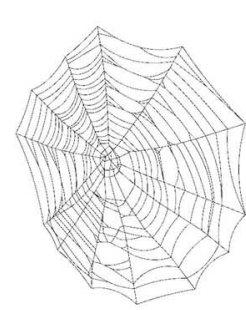

(A)

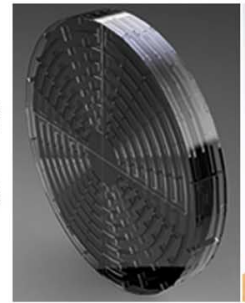

(B)

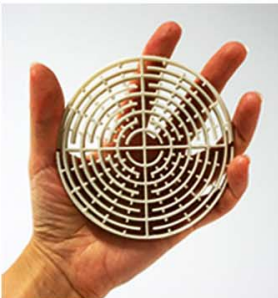

(C)

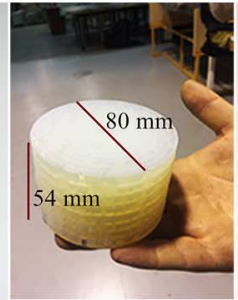

(D)

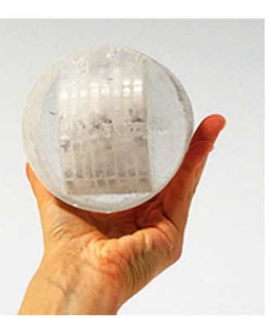

(E)

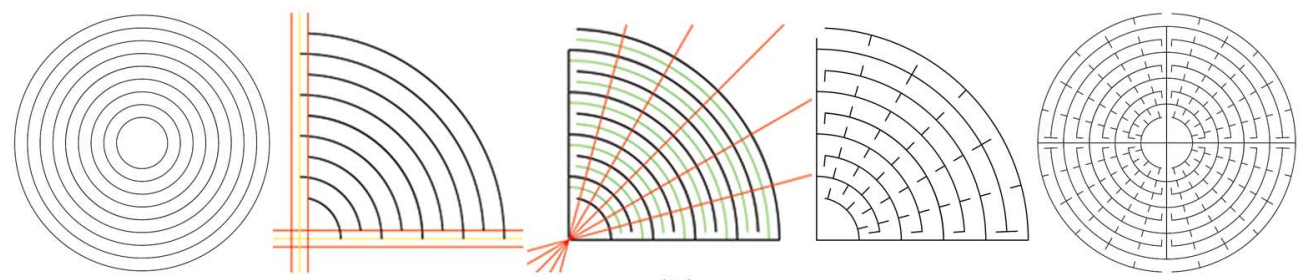

(F)

FIG. 3 Examples of $(A)$ DNA, $(B)$ synapse, and $(C)$ molecule structures reflecting the idea of biomimicry, (D) DNA module top view, (E,F) 3-D models of DNA module, (G) molded sample ready for measurement, and (H) a faulty 3-D print result.

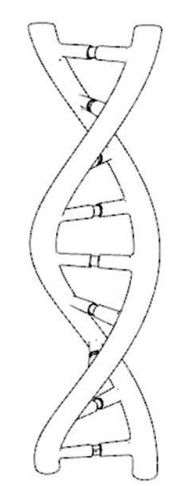

(A)

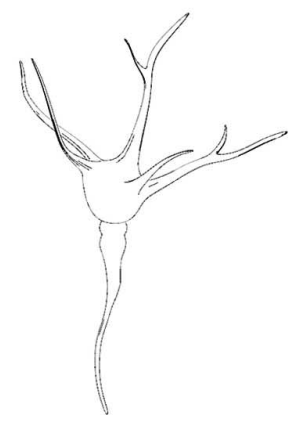

(B)

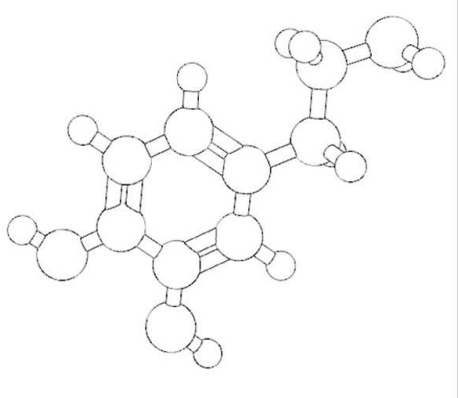

(C)

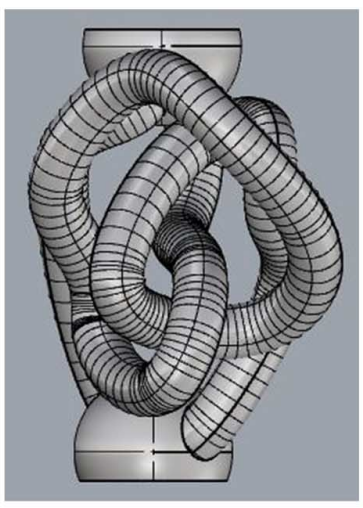

(D)

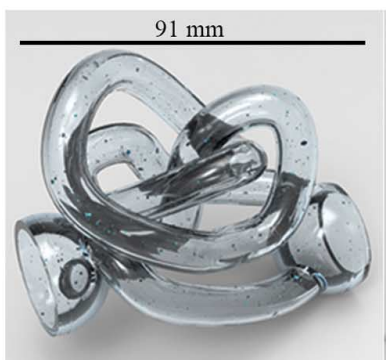

(E)

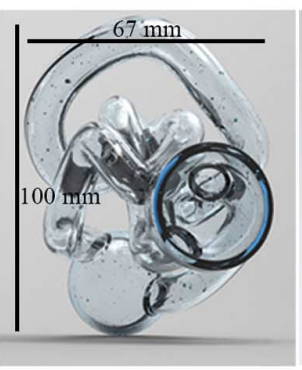

$(F)$

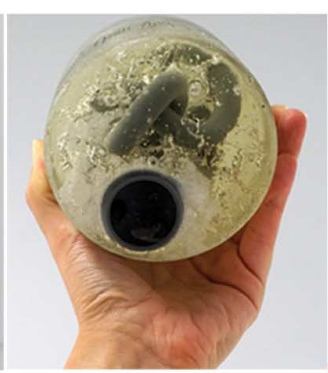

(G)

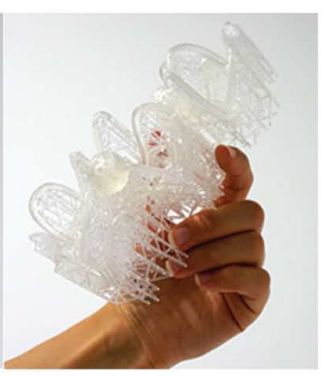

(H) 
of its geometry. When the spider's web, which is created in 2-D, is extrapolated to 3-D, the web itself becomes a mass, and the voids form the paths along which sound waves travel.

The abstraction of the WL proposal and the development of its design consist of the following steps. Firstly, a circle $8 \mathrm{~cm}$ in diameter is taken and offset $4 \mathrm{~mm}$ inward several times to create concentric circles. Secondly, onequarter of the circle is cut out. Rays are then introduced $15^{\circ}$ apart, and small masses are repeatedly placed along these rays in such a way that the distance between each mass in the same cavity with the corresponding mass in the same axis is $1.5 \mathrm{~mm}$. Then, this quarter-circle segment is mirrored to make a semicircular segment, and the semicircular piece is in turn mirrored to form the full circle back again. This constitutes the first layer of the WL module (fig. $2 F$ ).

Unlike the GR proposal, the WL unit has a multilayered structure. In total, there are four types of layers. Two of them have openings on the sides, and the other two have openings at the center. The openings enable each layer to connect with the two adjacent layers above and below it (fig. 2B). Two layers of the same type are not permitted to overlap: each layer must be different from the one below it and the one above it. The junctions in the central parts of the units are for extending the path and for coiling up the sound waves. There is no limitation on the number of times the layers can be repeated, so the depth of the module is adjustable. The 3-D-printed unit has a depth of $8 \mathrm{~cm}$, and there are eight layers with a total width of $5.4 \mathrm{~cm}$ (fig. 2D). The final output, with outer parts of the web filled with resin and ready to test in an impedance tube, has a diameter of $10 \mathrm{~cm}$ and a depth of $8 \mathrm{~cm}$ (fig. 2E). The length of inner void is $1,007 \mathrm{~cm}$.

\section{DNA MODULE DESIGN}

The third proposed AMM module is the DNA module. The idea is taken from natural transmission systems, such as the spiral structure of $\mathrm{DNA}^{37}$ that provides for the transfer of genes (fig. 3A). The spiral form serves to extend the path that the sound waves travel, as intended. The multidirectional/branched structure of synapses, ${ }^{38}$ which allow for the transmission of senses in the neural system (fig. 3B), is used in the sample AMM to divide the sound pathway into two tubes, thus further reducing its energy. This is why the DNA specimen has more than one tube. Finally, the semispherical parts of the DNA unit, with their apertures, have their origins in the spherical structure of the nodes that connect the particles in a molecule structure, ${ }^{39}$ including atomic particles (fig. $3 \mathrm{C}$ ). The specimen prepared for measuring in the impedance tube is $10 \mathrm{~cm}$ in diameter and $9.5 \mathrm{~cm}$ in depth (fig. 3G). The length of inner void is $66 \mathrm{~cm}$.

In the designed structure of the DNA module, as shown in figure 3, the openings on the opposite sides are approximately hemispherical. The tubes have a diameter of $10-13 \mathrm{~mm}$, as the manufacturing tool available for this piece of research is not sensitive in dimensions of less than $5 \mathrm{~mm}$. Because of the limited sensitivity of the equipment used, several unsatisfactory results were printed initially (fig. $3 H$ ). The design and manufacturing process continued until the diameter of the pipes could be optimized and a reasonable end product obtained.

\section{MANUFACTURING PROCESS}

The actual production of the designed modules depends on the available manufacturing technology. Manufacturing coiled-space materials that use forms like labyrinths and spirals present difficulties. Many challenges and limitations have been encountered in the fabrication of metamaterials, even when using modern technology that is capable of producing complicated forms. One problem is that a material designed to achieve a certain acoustic performance may not have the necessary practical or mechanical strength. The costs of the base materials and access to manufacturing tools may also constitute a challenge.

The voids or cavities inside the AMM modules shape the path of the sound, while the solid parts provide the necessary impedance. The cleanness or smoothness of the tubes in spiral and labyrinth patterns are critical for the coiling up of the voids and hence for the intended manipulation of the sound paths. It is therefore of great importance that the manufactured product should be an exact rendering of the design. Design optimization and production efforts continued until satisfactory end products were obtained for all three of the AMM modules developed in this study. 
FIG. 4 Molding phases: $(A)$ mixing epoxy with the required amount of dryer, $(B)$ vacuuming air to ensure a smooth inner surface, $(C)$ epoxy being poured into the 3-D printed inner structure and left to dry, and $(D)$ AMM prototype ready for measurement.

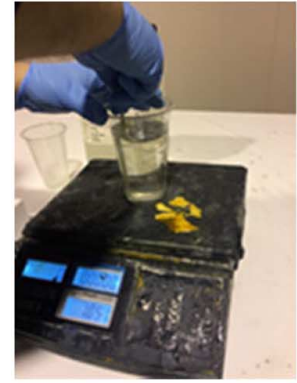

(A)

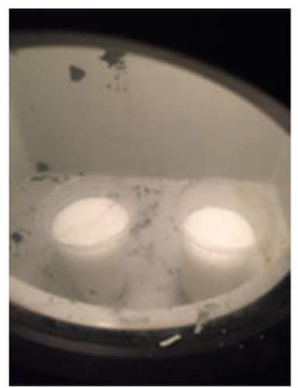

(B)

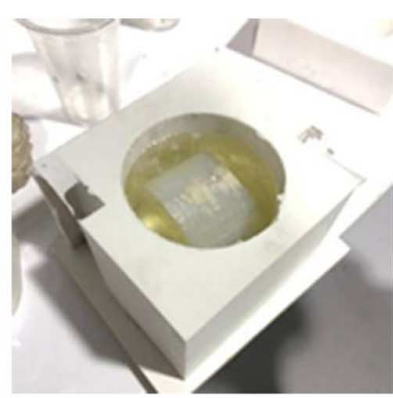

$(C)$

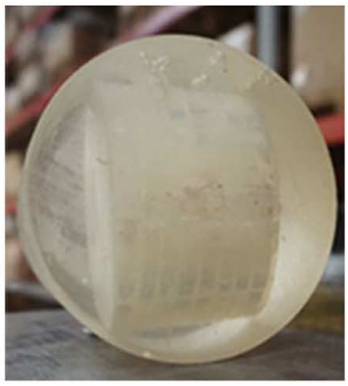

$(D)$

This study makes use of 3-D printing and additive manufacturing techniques to produce units of the proposed AMMs accurately. There are studies in room acoustics ${ }^{40}$ and many other fields that apply this method for in-depth scientific research with prototypes. ${ }^{41,42}$ During the manufacturing process, the inner structures (tubes or void walls) are initially produced by the 3 -D computer numerical control (CNC) printer. The AMM modules are finalized through the molding and filling of the outer cylinder to be fitted in the impedance tube. In an actual application, the inner structures would remain the same, while the rest of the filled form would be variable, depending on design decisions. Figure 4 presents the molding phases of the AMM production process. The main reason for molding the outer envelope of the cylindrical module is to be able to use a less expensive type of resin compared with that used in the 3-D printing.

After the first two molding phases (fig. $4 A$ and $4 B$ ), the module is left to dry at room temperature. The time required for drying at room temperature (fig. $4 \mathrm{C}$ ) is approximately $24 \mathrm{~h}$. If the whole process was completed by printer, the time taken by the process would be shorter. The 3-D CNC printer would take approximately $12 \mathrm{~h}$ to produce the full form ready for measurement in an impedance tube. However, the project budget would be too high with this method, so only the inner structure is printed using the 3-D CNC. This production technique could accelerate the process depending on the key factors of resolution, layer thickness, and printing strategy. ${ }^{43}$

\section{Acoustical Test and Assessment Methods}

In the empirical part of this research, the impedance tube method is held for testing the sound absorption and TL performance of AMM proposals. In the theoretical part, TL estimates on typical drywall systems are examined for comparison. Later, analytical assessments of proposed materials are held using a neckless Helmholtz resonator and PDI methods. The final part of this section represents a case study setup involving field measurements and room acoustics simulations.

\section{IMPEDANCE TUBE MEASUREMENTS AND TL ESTIMATES}

Once the proposed AMM modules have been produced, they are tested in an impedance tube (S.C.S. Kundt Impedance Tubes) to obtain sound absorption and sound TL values for 1/3 octave bands below 1,200 Hz. The absorption coefficient and acoustic impedance measurements are made using a two-microphone transfer function method in line with ISO 10534-2:1998, Acoustics - Determination of Sound Absorption Coefficient and Impedance in Impedance Tubes - Part 2: Transfer-Function Method, ${ }^{44}$ while the TL measurements are done using a four-microphone transfer function method. The AMM tests in this study are conducted using a 100-mmdiameter tube for measurements in the frequency range from 50 to 1,200 Hz. It should be noted that impedance 
tube measurements can only provide normal incidence absorption coefficients, whereas the reverberation room method can provide random incidence values. Both methods have their advantages and disadvantages. In metamaterial research, impedance tube tests are predominantly used, as they are more practical. That said, in the discussion of acoustical enhancement that follows, relative values rather than absolute values are compared for the states of the studios with solid reflective surfaces and with AMM surfaces.

The drawing (fig. 5) presents how the sound absorption coefficient measurements are done with the impedance tube method. As can be seen inside the tube (on the left side of the cavity), the depth of the cavity can be adjusted with the help of the linear apparatus behind the cylindrical plate. Therefore, the amount of air gap can be reduced or increased. Figure 5 shows the " $5 \mathrm{~cm}$ gap between AMM and tube plate" configuration. There may be no gap between the cylindrical plate and AMM samples, which refers to "two sides open, without gap" configurations. Also, specifically in the DNA module, the air gaps of 2.5, 5, 7.5, 10, and $12.5 \mathrm{~cm}$ behind the module are tested in sound absorption measurements. These tests are conducted to compare the performance of different air gaps behind the AMM panels for actual applications.

Table 1 presents the descriptions of the GR, WL, and DNA module configurations and the solid sample in the impedance tube for sound absorption $(\alpha)$ and TL $(\mathrm{dB})$ measurements. Table 1 also includes descriptions of a number of wall section configurations that are only theoretically estimated. Since the proposed AMMs are intended for architectural applications, the air space behind the modules is a critical variable. The openings in the rear sides of the modules are closed in some configurations to check whether there are any meaningful differences between their performance when both sides are open and when one side is closed. When the apertures on the rear sides of the samples are closed, the AMMs may act more like a Helmholtz resonator. ${ }^{14,26}$

\section{ANALYTICAL ASSESSMENT OF SOUND ABSORPTION COEFFICIENTS OF PROPOSED METAMATERIALS}

In this section, the analytical approaches in assessment of impedance tube measurement results of proposed AMMs are presented. The aim is to critically evaluate the possible diagnostic tools that can augment the design process and interpretation of output data in AMM design.

\section{NECKLESS HELMHOLTZ RESONATOR CALCULATIONS}

Studies ${ }^{45,46}$ have pointed out that AMMs may act like Helmholtz resonators in certain configurations. The internal structures of the three different AMM samples proposed in this study also have similarities with neckless Helmholtz resonators. To check whether the sound wave behavior can be explained by a neckless Helmholtz resonator function for certain types of proposed modules, theoretical calculations are held.

A special type of air-spring oscillator, a so-called Helmholtz resonator, is a volume with an opening at the one end and a small neck that is activated in its specific resonant frequency. Using the relationship shown in figure 6 , the resonant frequency of the system can be calculated by equation $(1)^{47}$ :

FIG. 5

Sound absorption coefficient measurement setup: abstracted 3-D model of impedance tube indicating AMM sample locations under different configurations (prepared by the author).

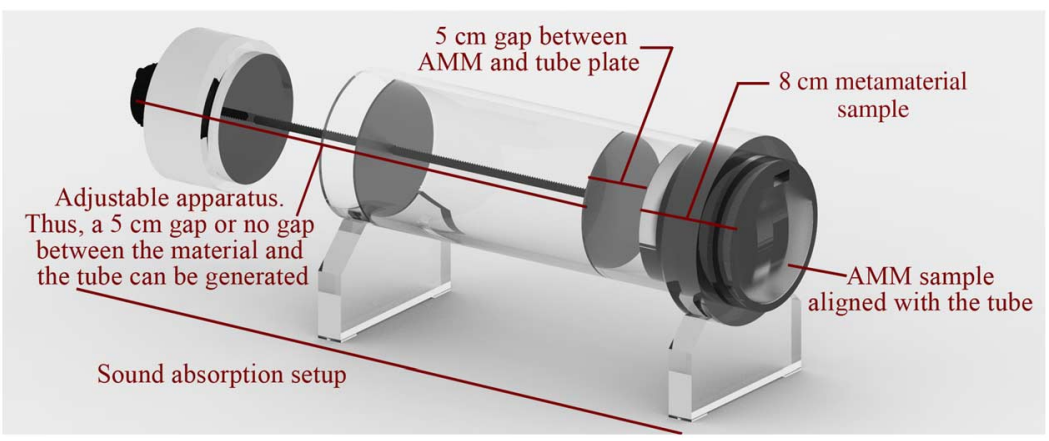




\section{TABLE 1}

Configurations of the material samples tested by impedance tube and used in theoretical transmission (TL) estimates

\begin{tabular}{ll}
\hline Code of Sample & \multicolumn{1}{c}{ Description } \\
\hline SS1 & $8 \mathrm{~cm}$ (resin) solid sample $+5 \mathrm{~cm}$ gap \\
SS2 & $8 \mathrm{~cm}$ (resin) solid sample without gap \\
SS3 & $8 \mathrm{~cm}$ (resin) solid sample (for TL measurement) \\
GR1 & $8 \mathrm{~cm} \mathrm{GR,} \mathrm{two} \mathrm{sides} \mathrm{open}+5 \mathrm{~cm}$ gap \\
GR2 & $8 \mathrm{~cm} \mathrm{GR,} \mathrm{one} \mathrm{side} \mathrm{closed}+5 \mathrm{~cm}$ gap \\
GR3 & $8 \mathrm{~cm} \mathrm{GR,} \mathrm{two} \mathrm{sides} \mathrm{open,} \mathrm{without} \mathrm{gap}$ \\
WL1 & $8 \mathrm{~cm}$ WL, two side open $+5 \mathrm{~cm}$ gap \\
WL2 & $8 \mathrm{~cm}$ WL, one side closed $+5 \mathrm{~cm}$ gap \\
WL3 & $8 \mathrm{~cm}$ WL, two sides open, without gap \\
DNA1 & $8 \mathrm{~cm} \mathrm{DNA,} \mathrm{two} \mathrm{sides} \mathrm{open}+5 \mathrm{~cm}$ gap \\
DNA2 & $8 \mathrm{~cm} \mathrm{DNA,} \mathrm{one} \mathrm{side} \mathrm{closed}+5 \mathrm{~cm}$ gap \\
DNA3 & $8 \mathrm{~cm} \mathrm{DNA,} \mathrm{two} \mathrm{sides} \mathrm{open,} \mathrm{without} \mathrm{gap}$ \\
DNA4 & $8 \mathrm{~cm} \mathrm{DNA,} \mathrm{two} \mathrm{sides} \mathrm{open}+2.5 \mathrm{~cm}$ gap \\
DNA5 & $8 \mathrm{~cm} \mathrm{DNA,} \mathrm{two} \mathrm{sides} \mathrm{open}+7.5 \mathrm{~cm}$ gap \\
DNA7 & $8 \mathrm{~cm} \mathrm{DNA,} \mathrm{two} \mathrm{sides} \mathrm{open}+10 \mathrm{~cm}$ gap \\
DNA6 & $8 \mathrm{~cm} \mathrm{DNA,} \mathrm{two} \mathrm{side} \mathrm{open}+12.5 \mathrm{~cm}$ gap \\
Y1 & $2 \mathrm{~cm}$ resin panel, $4 \mathrm{~cm}$ gap, $2 \mathrm{~cm}$ resin panel \\
Y2 & $1 \mathrm{~cm}$ resin panel, $6 \mathrm{~cm}$ gap, $1 \mathrm{~cm}$ resin panel \\
Y3 & $1 \mathrm{~cm}$ resin panel, $6 \mathrm{~cm}$ mineral wool $\left(48 \mathrm{~kg} / \mathrm{m}^{3}\right), 1 \mathrm{~cm} \mathrm{resin} \mathrm{panel}$ \\
Y4 & $1 \mathrm{~cm}$ resin panel, $5 \mathrm{~cm}$ gap, $2 \mathrm{~cm}$ resin panel \\
\hline &
\end{tabular}

FIG. 6

Conceptual drawing of a neckless Helmholtz resonator.

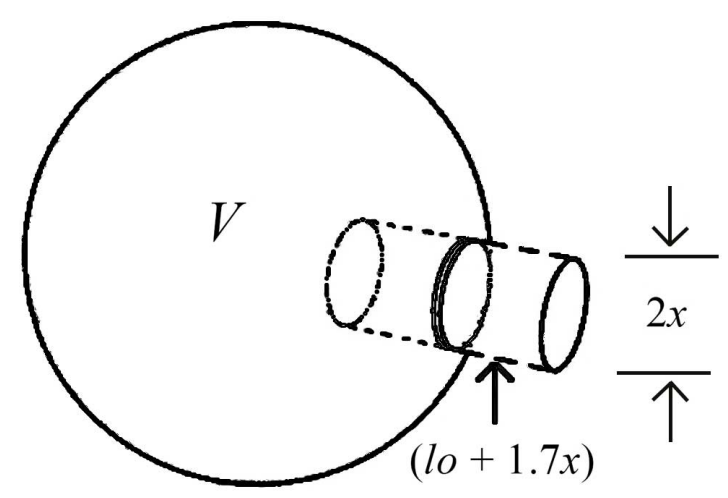

$$
f_{n}=\frac{c_{0}}{2 \pi} \sqrt{\frac{\pi x^{2}}{V\left(l_{0}+1.7 x\right)}}
$$

where $f_{n}$ is the natural frequency of the system, $c_{0}$ is the speed of the sound in the air, $V$ is the enclosed volume, $x$ is the radius of the opening, and $l_{0}$ is the thickness of the plate. Equation (1) is utilized in interpretation of the results as detailed in the following sections.

\section{PDI CALCULATIONS}

As a second analytical method, PDI is included in this study since AMMs can also dampen sound waves as they pass through the structure by creating destructive interference. PDI can occur where the sound waves combine 
with a phase difference of $180^{\circ}$, or half of the wavelength, and therefore neutralize each other. This method uses the sound itself to greatly reduce its amplitude. Passive means that active sound sources, such as speakers, are not used. ${ }^{33}$ Figure 7 illustrates the basic principle of the Herschel-Quincke Tube, ${ }^{48}$ which has a similar form to a PDI absorber along an airway of a certain length, in which incoming sound waves enter from either one or both sides.

There is a relation between the length of air path and the frequency at which the peak value of sound absorption coefficient of PDI is detected. As shown by equation (2), the value of $n$ is a positive integer $(1,2,3,4 \ldots)$, $f(\mathrm{~Hz})$ is the frequency, $c(\mathrm{~m} / \mathrm{s})$ is the speed of sound in air, and $\Delta L(\mathrm{~m})$ is the length difference of the transmission. The transmission length difference $\Delta L$ relates to the location of the interference point. According to Setaki et al.'s study, ${ }^{33}$ this interference occurs above one quarter of the air path and below the total length of it. In equation (3), $\Delta L$ is represented as a set of limits that anticipates the frequencies at which destructive interference may occur, as shown in equation (4). PDI equations are utilized to inspect the applicability of the method for different AMM modules developed in this study:

$$
\begin{gathered}
f=\frac{(2 n-1) c}{2 \Delta L} \\
\frac{L_{t o t}}{2}<\Delta L<L_{t o t} \\
\frac{(2 n-1) c}{2 L_{t o t}}<f<\frac{(2 n-1) c}{L_{t o t}}
\end{gathered}
$$

\section{CASE STUDY SETUP}

The final step in the study involves testing the performance of the proposed materials in a real design problem through the use of room acoustics simulations. For this purpose, two design studios are selected that currently have no acoustical interventions. In shared rooms like studios, open-plan offices, or restaurants, noise control is an important comfort parameter, but interior design is also a significant concern. To attenuate the high noise levels in the studios at low frequencies resulting from the highly reflective painted brick and concrete surfaces, some of the proposed AMM units are developed into wall partitions (panel systems) with a modular layout and two different heights. These partitions are then placed between the drawing tables. Also taken into consideration here is the fact that these translucent, resin-based AMMs can be in different color combinations, some of which better highlight the inner structure of the AMM designs (fig. 8D) and can give a rhythm to the studio space.

It has previously been demonstrated in practice that when metamaterials are placed in a repeating lattice structure, ${ }^{5,6,49,50}$ such as in the form of origami folding, ${ }^{51}$ the efficiency of the material structure increases and

FIG. 7 Acoustic pressure circulation of an open-open pipe and a closed-open pipe along an airway of a certain length ( $L$ ). Pressure antinodes shown as $\mathrm{AN}$ and pressure nodes shown as $\mathrm{N}$.

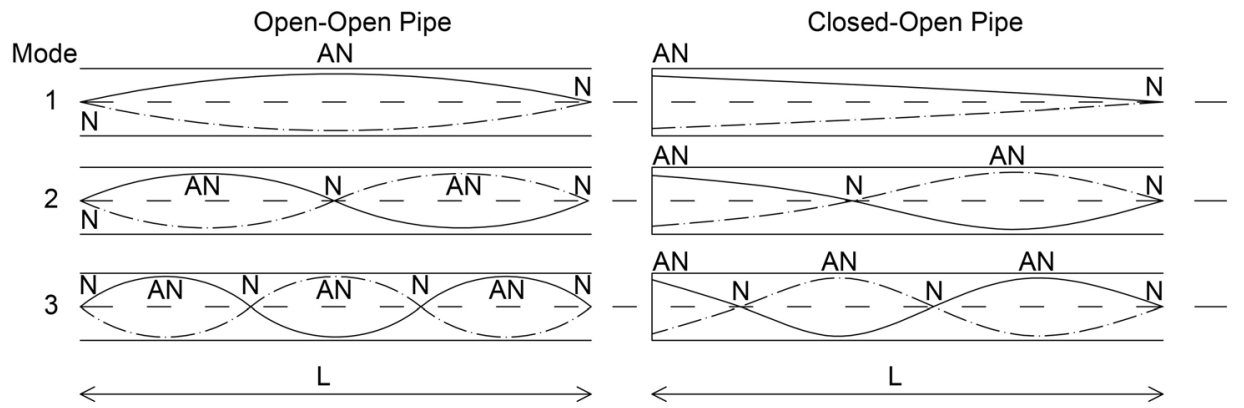


FIG. 8 (A) Studio\#2 3-D model with design proposal using 1/5 height AMM panels, (B) Studio\#2 with design proposal using 3/4 height AMM panels, (C) 3-D models showing sound source and receiver positions in Studio\#1, (D) panels with DNA5 on one side and WL on the other, with a $7.5 \mathrm{~cm}$ gap in between, shown in Studio\#1 and Studio\#2, and

(E) photograph of the field tests in Studio\#1.

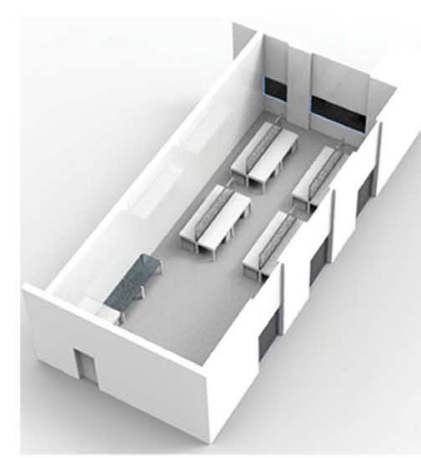

(A)

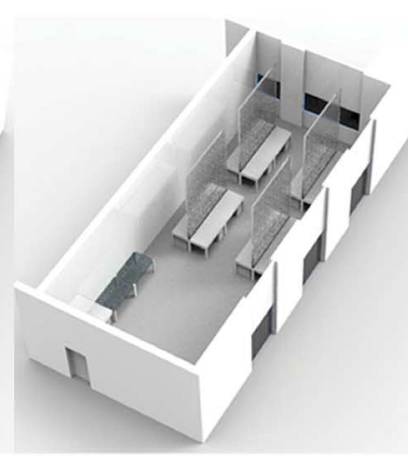

(B)

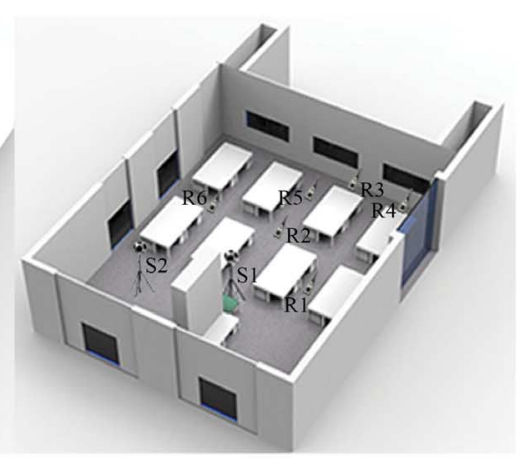

(C)

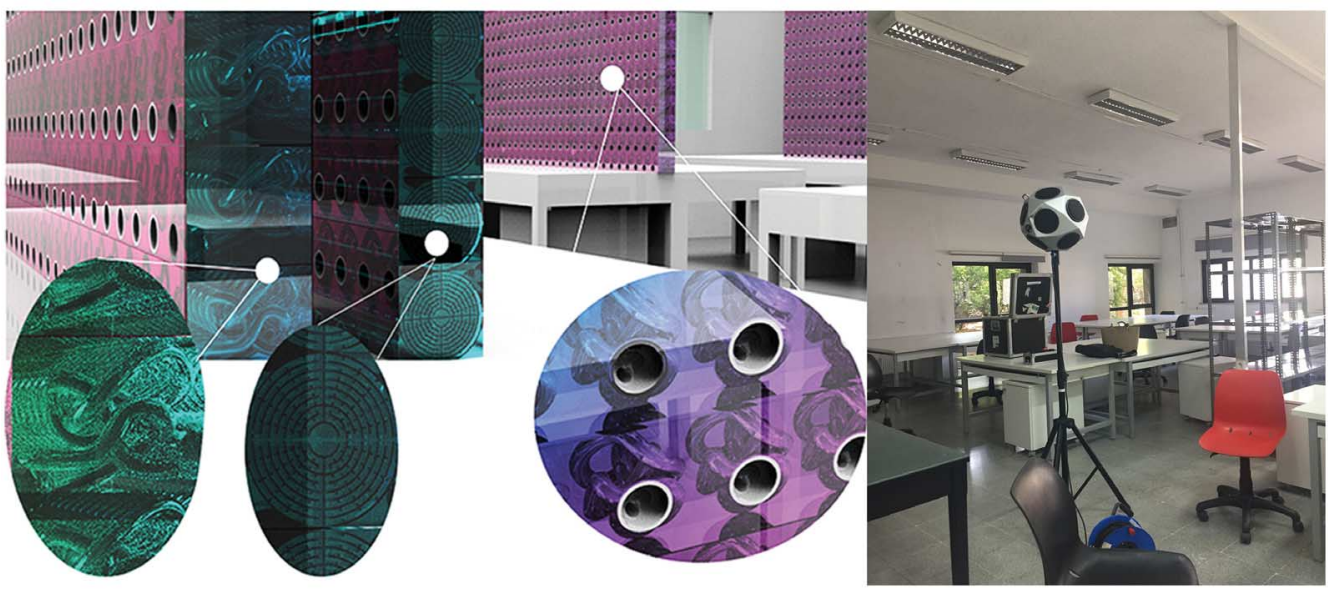

(D)

(E)

a better performance is obtained. To estimate the acoustic performance of the AMM modules, the studios are first field-tested to determine their current acoustic parameters. Then, the acoustical models are generated and tuned according to the field test data for further experimental study, as described in the following.

\section{FIELD MEASUREMENTS}

The field tests of the studios were held during the summer break in the academic year, on August 6th, 2019, between the hours of 12:30 and 14:00. The studios are empty and with minimum background noise conditions (fig. 8E). To estimate the basic parameters specifying the sound field, the room impulse responses are collected. The equipment used is in accordance with ISO 3382-2:2008 Acoustics - Measurement of Room Acoustic Parameters - Part 2: Reverberation Time in Ordinary Rooms Standard. A B\&K (Type 4292-L) dodecahedron omnidirectional sound source is used for acoustic excitation, with a B\&K (Type 2734-A) power amplifier. The room impulse responses at various measurement points are captured by a B\&K (Type 4190ZC-0032) microphone. The sampling frequency of the recorded multispectrum impulse is $48 \mathrm{kHz}$. The height of the omnidirectional sound source is $1.5 \mathrm{~m}$ from the floor, and the microphone height is maintained at $1.2 \mathrm{~m}$. DIRAC Room Acoustics Software Type 7841 v.4.1 is used to generate e-sweep noise signals. Two source and six receiver locations are used for Studio\#1 (fig. 8C), and two source and seven receiver locations are used for Studio\#2. These 
numbers are considered to be sufficient given the dimensions of the studios. The acoustical metrics, particularly the reverberation times (T20 and T30), obtained over the postprocessed impulse responses are utilized for tuning the materials assigned in the acoustical models, as described in the following paragraphs.

\section{ROOM ACOUSTICS SIMULATIONS}

The aim of the room acoustics simulations is to assess the relative improvement that can be achieved in an architectural application of the proposed AMMs by comparing values before and after the intervention rather than to obtain the absolute acoustic parameter results of the room impulses. In this process, simplified 3-D graphical models of the existing studios (Studio\#1 and Studio\#2) were initially developed for use with ODEON Room Acoustics Software version 14.04. As shown in figure 8, Studio\#1 has a capacity of 32 students and an estimated acoustic volume of $\sim 997 \mathrm{~m}^{3}$. The student capacity for Studio\#2 is 16 , and it has an estimated acoustic volume of $\sim 526 \mathrm{~m}^{3}$. The study focuses on T30 values at 63, 125, 250, 500 and 1,000 Hz, SPL (A) (A-weighted sound pressure level), and speech transmission index (STI) results. For the STI grid results, the sources are positioned $1.20 \mathrm{~m}$ off the floor, and the distance between receivers is $0.4 \mathrm{~m}$. In the analysis of speech intelligibility in the proposed scenario, the background noise level is assumed not to exceed the Noise Criteria 30 (NC30) level.

Simulations are run for the current condition of the studios and for two different scenarios in which the proposed AMMs are applied for acoustical enhancement (fig. $8 A$ and $8 B$ ). The difference between the results of the simulations of the empty studios and the results of the field tests for T30 over tested receiver positions is around $0.03 \mathrm{~s}$, which is smaller than 1 just noticeable difference. This shows that the tuning of the acoustical model is sufficiently accurate.

Figure $8 A$ and $8 B$ show the positions and heights of the proposed partitions, built out of AMM modules, in Studio\#2. In Studio\#1, the locations of the partitions are the same, i,e., between each pair of drawing tables. The heights of the partitions are also the same. The proposal in which the partitions are $1 / 5$ of the height of the studio (fig. $8 A$ ) is referred to as Proposal A1, and the proposal in which the partitions are $3 / 4$ of the height of the studio (fig. $8 B$ ) is designated Proposal A2. The interior finish materials and the corresponding absorption coefficients obtained in the simulations are listed by frequency in Table 2.

\section{Results and Discussion}

\section{IMPEDANCE TUBE TEST RESULTS}

All three prototypes and solid samples are tested in an impedance tube with the different configurations listed in Table 1. Each configuration is tested a minimum of 10 times to ensure the replicability and reliability of the results. The sound absorption coefficients $(\alpha)$ and TL values in $\mathrm{dB}$ over $1 / 3$ octave bands below $1,200 \mathrm{~Hz}$ are presented in the following paragraphs.

TABLE 2

List of materials and sound absorption coefficients over 1/1 octave bands from 63 to 1,000 Hz

\begin{tabular}{|c|c|c|c|c|c|c|}
\hline Material Location & Name & $63 \mathrm{~Hz}$ & $125 \mathrm{~Hz}$ & $250 \mathrm{~Hz}$ & $500 \mathrm{~Hz}$ & $1,000 \mathrm{~Hz}$ \\
\hline Wall surfaces & Paint and plaster on brick wall & 0.06 & 0.06 & 0.06 & 0.10 & 0.10 \\
\hline Ceiling surfaces & Painted concrete & 0.10 & 0.10 & 0.05 & 0.06 & 0.07 \\
\hline Floor surfaces & Concrete or terrazzo flooring & 0.01 & 0.01 & 0.01 & 0.02 & 0.02 \\
\hline Drawing table & Wood boards on a profile & 0.15 & 0.15 & 0.20 & 0.10 & 0.10 \\
\hline Windows & Ordinary window glass & 0.35 & 0.35 & 0.25 & 0.18 & 0.12 \\
\hline Door & Wood, 1 layer, $20 \mathrm{~mm}$ & 0.25 & 0.25 & 0.18 & 0.11 & 0.08 \\
\hline Studio\# 1 & Corrugated sheet, foam-like plastic material, etc. & 0.30 & 0.30 & 0.45 & 0.65 & 0.56 \\
\hline Proposed module $1^{\mathrm{a}}$ & DNA 5 AMM, DNA with $7.5 \mathrm{~cm}$ air gap behind & 0.77 & 0.31 & 0.12 & 0.07 & 0.18 \\
\hline Proposed module $2^{\mathrm{a}}$ & $\mathrm{WL}$ & 0.03 & 0.03 & 0.02 & 0.06 & 0.06 \\
\hline
\end{tabular}

Note: ${ }^{\text {a }}$ Sound absorption coefficient values are obtained from impedance tube measurement results. 


\section{SOUND ABSORPTION COEFFICIENT MEASUREMENT RESULTS}

The sound absorption measurement results of the sample GR, WL, and DNA units are compared in figure 9. Among the various GR configurations tested, GR1 shows the least efficient sound absorption performance (fig. 9A). All the GR configurations perform better than the solid samples SS1 and SS2. Peak absorption is detected in the range of $100-125 \mathrm{~Hz}$, at $400 \mathrm{~Hz}$, and at $630 \mathrm{~Hz}$.

The sample WL unit demonstrates the lowest performance (fig. 9B). Its performance tends to parallel the reflectivity of the base material. The configuration WL2 has a peak absorption of 0.07 at $500 \mathrm{~Hz}$, and WL3 has a peak absorption of 0.05 at $80 \mathrm{~Hz}$ with a value of 0.05 . However, neither sample offers any notable advantage in comparison with the solid sample. Thus, the design parameters of the WL module need to be improved and optimized for better performance in sound absorption.

The sample DNA unit shows more potential for sound absorption, as presented in figure 9C. In particular, the DNA1 configuration (two sides open, $5 \mathrm{~cm}$ air gap behind) achieves sound absorption coefficient values up to 0.70 at $80 \mathrm{~Hz}$, which is remarkably above average. By contrast, the solid (unperforated) samples, SS1 and SS2, have absorption coefficients in the range of $0.02-0.03$. The only difference between DNA2 and DNA1 is that the opening on the rear surface is closed. This difference causes DNA2 to achieve the least effective sound absorption result. In other words, the aperture on the rear surface is of great importance for increasing the sound absorption efficiency of the DNA module. The DNA3 configuration (two sides open, no air gap behind), has higher sound absorption values at frequencies between 250 and $315 \mathrm{~Hz}$.

Since DNA1 is the best sound absorber among the alternatives tested, additional configurations are developed by changing the size of the gap between the tube and the DNA AMM module. The measurements were performed again for the "two sides open" variation of the DNA module with air gaps of different dimensions behind the module (fig. 9D).

As can be observed from figure 9D, configuration DNA5 (2 sides open, $7.5 \mathrm{~cm}$ air gap) achieves its maximum sound absorption value at $63 \mathrm{~Hz}$ with an absorption coefficient of 0.77 . However, DNA1 still performs better than the other configurations across the tested frequency spectrum as a whole. For all DNA types, sound

FIG. 9 Measured sound absorption coefficients over $1 / 3$ octave bands ( $\mathrm{Hz}$ ) for the following configurations of the AMM modules tested with the impedance tube: (A) GR1, GR2, GR3, SS1, and SS2; (B) WL1, WL2, WL3, SS1, and SS2; (C) DNA1, DNA2, DNA3, SS1, and SS2; and (D) DNA4, DNA1, DNA5, DNA6, DNA7, and SS1.

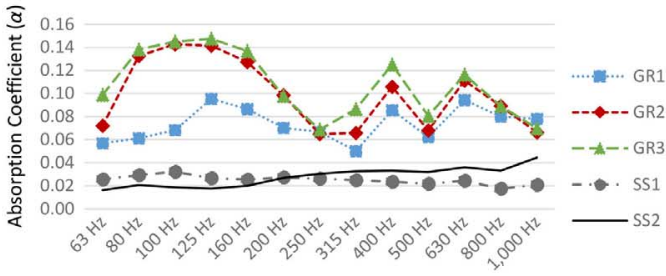

(A)

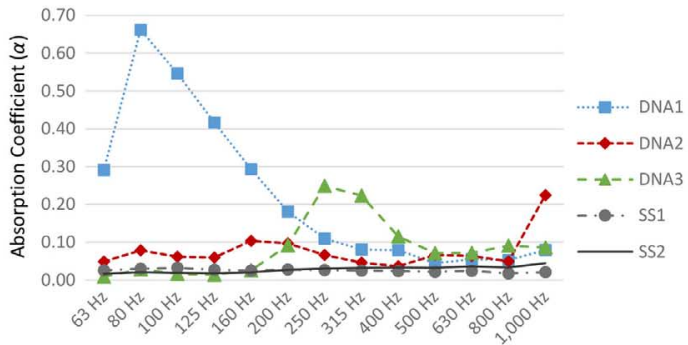

(C)

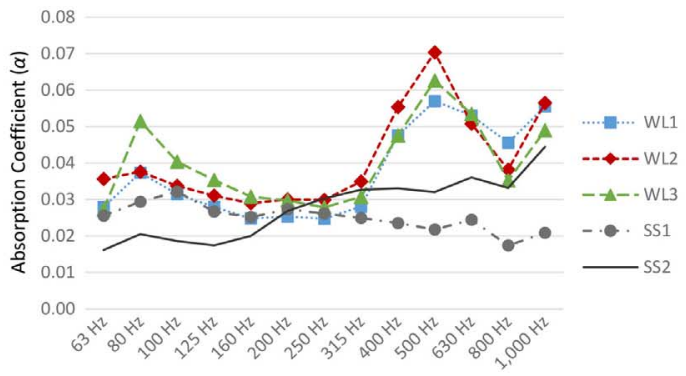

(B)

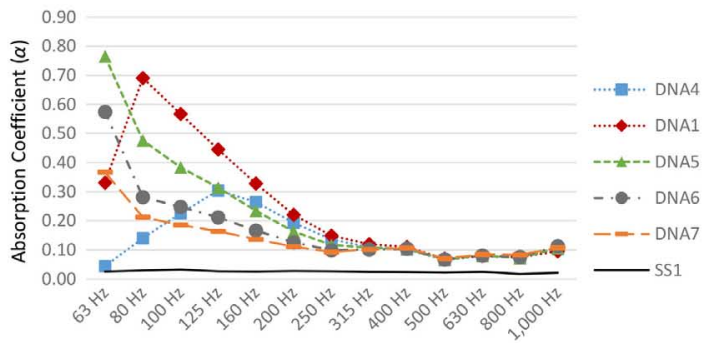

(D) 
absorption is greater in the $63-100 \mathrm{~Hz}$ frequency range than in the octave bands above $250 \mathrm{~Hz}$, in which it shows a pattern that is a little different from the solid sample. To conclude, the DNA module in its current state offers an effective sound absorption mechanism in a range of 63 to $200 \mathrm{~Hz}$. The peak frequencies, or the effective region on the frequency spectrum, can be adjusted by optimizing the design parameters/dimensions of the internal tubes.

\section{TL MEASUREMENT RESULTS}

The TL measurement results of the GR, WL, and DNA modules are compared in figure 10A. In contrast with their poor sound absorption performance, the sample WL unit is much more effective than the other proposed AMMs with respect to sound TL. For the frequency range below $1,200 \mathrm{~Hz}$, the TL values for WL (approximately 48-55 dB) are significantly higher than the results for the DNA and GR modules. The TL values of the solid sample SS3 range from 35 to $43 \mathrm{~dB}$ in the frequency interval of $125-160 \mathrm{~Hz}$, whereas the TL values of the WL configurations range from 48 to $55 \mathrm{~dB}$. Thus, the TL values of the WL unit are on average $12 \mathrm{~dB}$ higher than the values found for SS3. Meanwhile, the GR module provides approximately the same TL values as the solid sample SS3 since the inner structure of GR module is not efficient for sound transmission performance. The sample DNA unit has the least sound TL performance of all. On the contrary, the DNA proposal has more potential for sound absorption, as shown previously in figure $9 C$ and $9 D$.

Overall, these results suggest that the DNA and GR units are not suitable for use as a noise barrier in their current form but can be utilized in room acoustics for sound absorption. The WL proposal has more potential for use in noise control applications and can therefore be considered for use in practice as a separating wall/partition between two rooms/spaces (this was tested in the case study).

As part of the impedance tube tests, theoretical TL estimates are also made for typical lightweight walls using the same base material (epoxy resin) as the proposed AMMs (fig. 10B). The purpose of making these estimates is to discuss the effectiveness of the TL performances of the sample AMMs in comparison with those of drywall

FIG. 10

$T L$ measurement results of (A) WL, GR, DNA, and SS3 samples over $1 / 3$ octave bands $(\mathrm{Hz})$ below $1,200 \mathrm{~Hz}$ and $(B) \mathrm{WL}$ in comparison with theoretically estimated Y1, Y2, Y3, Y4, and SS3.

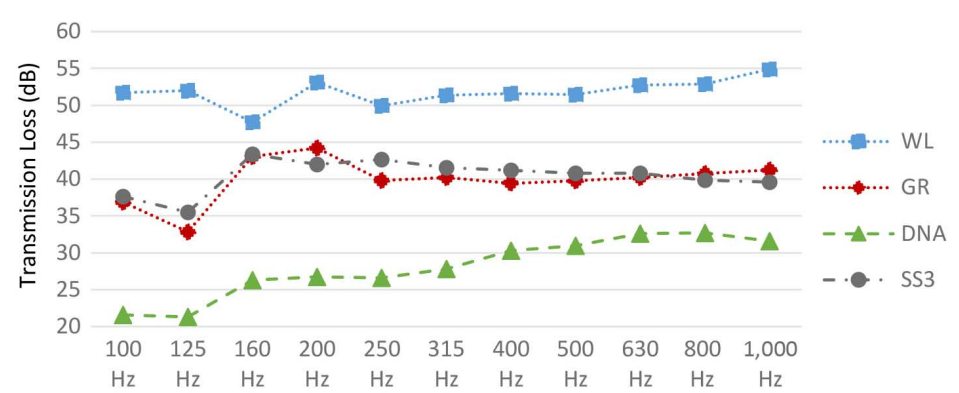

(A)

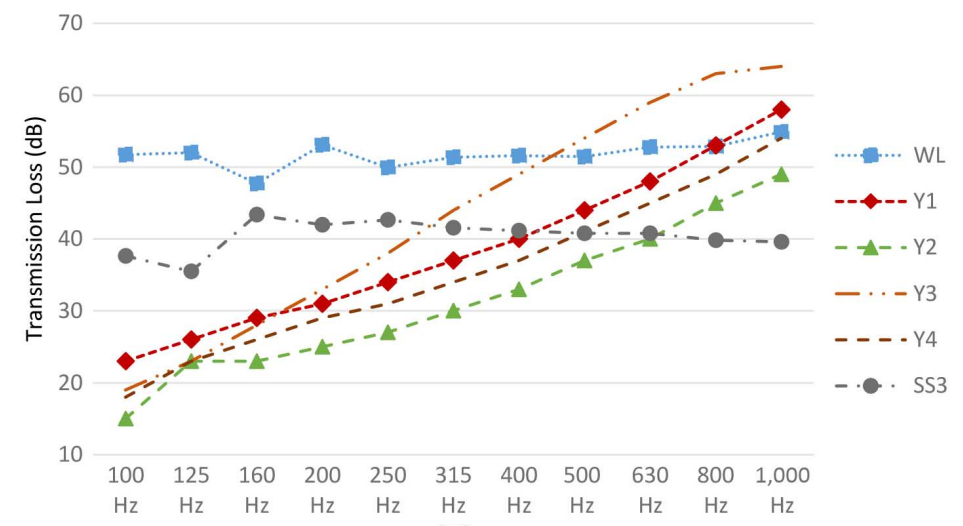

(B) 
systems with an air gap. Estimates are made both for wall sections in which the air gap is left unfilled and for wall sections in which a fibrous material is included within the air gap. This makes it possible to compare the performances of the proposed AMMs with the performances of conventionally used wall sections, such as those with a mineral wool infill. The density of epoxy resin is taken to be $1,098 \mathrm{~kg} / \mathrm{m}^{3}$.

To this end, the epoxy resin material is shaped into modules with standard configurations that are considered to exemplify the real wall applications (see Table 1 for cross-section definitions). The impedance tube results obtained from these modules are presented and compared with the results for WL and a solid sample in figure 10B. The total thickness of each wall section module is $8 \mathrm{~cm}$, the same as for the AMM modules. The WL module has the best TL performance overall. Above $500 \mathrm{~Hz}$, the Y3 configuration $(1 \mathrm{~cm}$ resin panel, $6 \mathrm{~cm}$ mineral wool of $48 \mathrm{~kg} / \mathrm{m}^{3}, 1 \mathrm{~cm}$ resin panel) generates higher TL values than the other wall section configurations and the WL module. The Y1 configuration ( $2 \mathrm{~cm}$ resin panel, $4 \mathrm{~cm}$ air gap, $2 \mathrm{~cm}$ resin panel) performs better than the $\mathrm{Y} 2$ configuration ( $1 \mathrm{~cm}$ resin panel, $6 \mathrm{~cm}$ air gap, $1 \mathrm{~cm}$ resin panel). The mineral wool filling used within the air cavity is the main reason for the higher performance of the $\mathrm{Y} 3$ configuration. However, this study pays specific attention to the use of nonfibrous and semitranslucent to transparent materials.

\section{RESULTS AND DISCUSSIONS OF ANALYTICAL ESTIMATES}

In this section, the analytical estimate methods are compared with the impedance tube measurement results. The analytical analysis, including the comparison of Helmholtz equation and PDI to measured data, is important to examine their applicability in the design stage of AMMs. In figure 11, the results obtained from the Helmholtz equation and PDI estimates are compared with the tube-measured results in the same graph.

In the Helmholtz equation estimates of the DNA sample, initially, one of the inner pipes is taken into consideration, and the resonant frequency is estimated to be $2,164 \mathrm{~Hz}$. Later, both inner pipes are included in the estimates, and the resonant frequency is calculated to be $848 \mathrm{~Hz}$. The impedance test results of the DNA module indicate that the peak absorption occurs between 63 and $80 \mathrm{~Hz}$, which is comparatively much lower than the estimated resonance frequencies. The Helmholtz equation estimates for the GR sample indicate a resonant frequency of $788 \mathrm{~Hz}$ and show a resonant frequency of $298 \mathrm{~Hz}$ for the WL sample. Therefore, none of the AMM prototypes in this research can be explained with Helmholtz resonator equations (fig. 11).

The second set of comparison is held for PDI estimates, designating the possible interval for the resonant frequency at which the absorption peaks can be observed. As shown in figure 11, the peak absorption range for GR is between 112 and $224 \mathrm{~Hz}$; for DNA, the range is between 261 and $523 \mathrm{~Hz}$; and for WL, the range is between 462 and $925 \mathrm{~Hz}$. On the other hand, the peak sound absorption coefficients in tube measurements are obtained at

FIG. 11 PDI and Helmholtz equation estimate results of GR, DNA, and WL in comparison with the results of impedance tube measurements for all three samples.

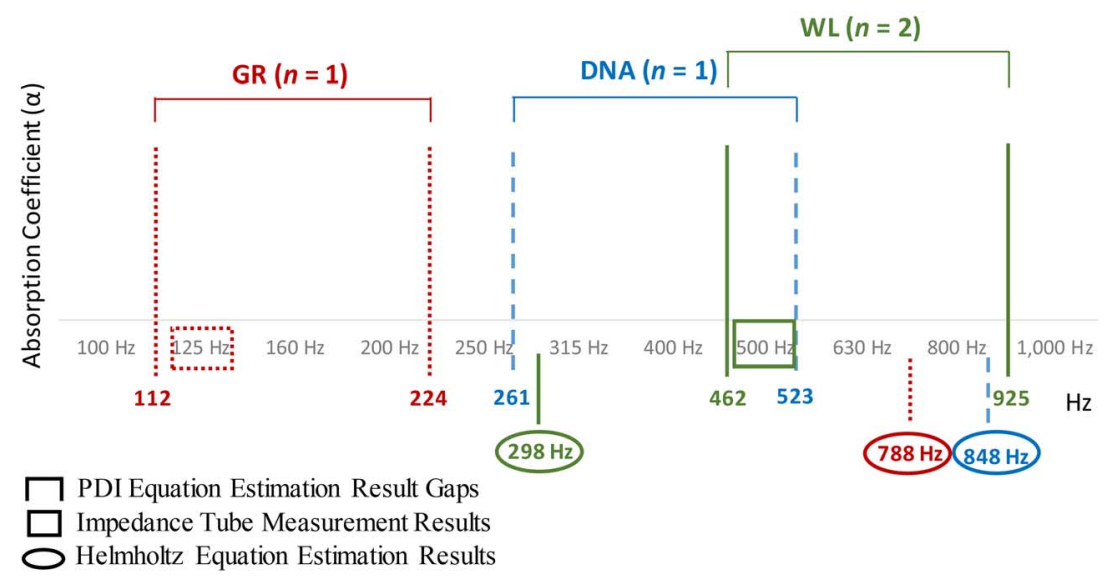


$125 \mathrm{~Hz}$ for GR, $63 \mathrm{~Hz}$ for DNA, and $500 \mathrm{~Hz}$ for WL. When the distribution of the sound absorption coefficients over $1 / 3$ octave bands for the GR module is analyzed, the frequency range with the highest results coincides with the results of the PDI calculations, which is between 63 and $200 \mathrm{~Hz}$. The measurement and the PDI estimates are coherent for GR and WL samples, which provides an initial guess of the frequency range during design phases. On the other hand, the DNA module cannot be explained either by the Helmholtz or the PDI method. This is due to the complexity of the inner voids, which cannot be simplified for the input parameters of the two analytical methods. For this reason, numerical studies on the DNA module are necessary in the future optimization phase. In a finite element medium, such a study will utilize the experimental data from this research. The present data will be used for tuning the complex Young's modulus characteristics of base material with and without the embedded structures for obtaining attenuation characteristics of the resin. Therefore, other alternative designs can be tested theoretically as well.

\section{ASSESSMENT OF PROPOSED AMM APPLICATION FOR THE ACOUSTICAL ENHANCEMENT OF DESIGN STUDIOS}

As detailed in the section "Acoustical Test and Assessment Methods," a case study was conducted in two studios, Studio\# 1 and Studio\#2, using field tests and simulations to assess the likely efficiency of the proposed AMMs in a real-life situation. The T30 values obtained from field measurements and the acoustical simulation results for the current condition of the studios and under the proposed acoustical intervention are given in figure 12. The T30 results for the studios in their current condition indicate a high level of reverberance of up to $2 \mathrm{~s}$ in both studios, far above the recommended limits for learning and studying environments, which should be $1 \mathrm{~s}$ at most for the given sizes of the studios. As previously noted, the control of low-frequency sound buildup within enclosed spaces, especially in a case like this with highly reflective massive wall and floor surfaces, is always challenging.

The proposed partitions are composed of DNA5 units, which achieved the highest sound absorption, on one face, and WL units, which achieved the highest TL values, on the reverse face. The acoustical simulations make use of the absorption coefficients and TL values obtained from the impedance tube measurements for the units in question.

FIG. 12

T30 values of

(A) Studio\#1 and

(B) Studio\#2 over octave

bands from 63 to

$1,000 \mathrm{~Hz}$, comparing

measured current

results, simulated current

results without AMM

panels, simulated results

after installing $1 / 5$ height

partitions (A1), and

simulated results after

installing $3 / 4$ height

partitions (A2).

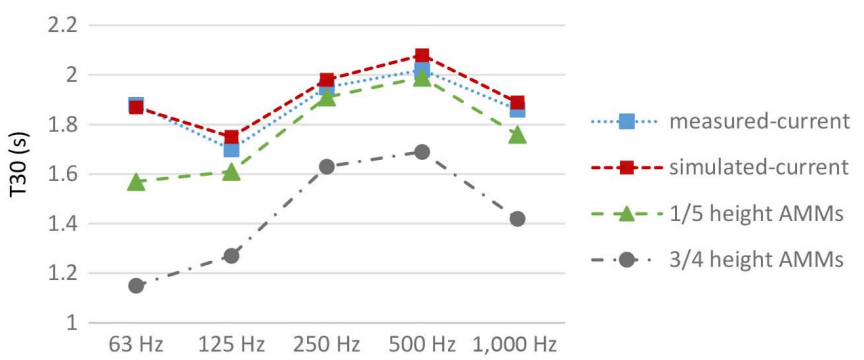

(A)

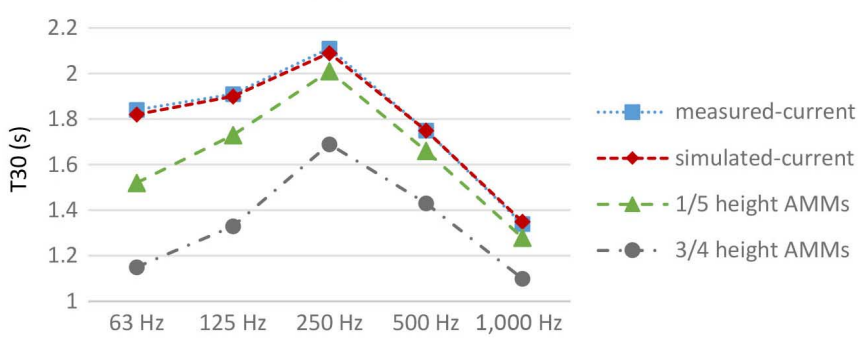

(B) 
In Proposal A1 (1/5 height partition), the T30 values improve by about $0.14-0.30 \mathrm{~s}$ in the range of $63-$ $125 \mathrm{~Hz}$. In frequency ranges above $125 \mathrm{~Hz}$, the T30 values gradually approach the readings for the current condition of the studios. In Proposal A2 (3/4 height partition), the improvement is much more significant, with T30 values varying between 1.1 and $1.69 \mathrm{~s}$ across the tested range of 63 to $1,000 \mathrm{~Hz}$. At $125 \mathrm{~Hz}$, for example, T30 shows a decrease of $0.43 \mathrm{~s}$ in Studio\#1 by comparison with the current condition of the studio. This is a significant improvement for this frequency range, particularly given the volume of the studio (fig. 12A).

Studio\#2 shows a similar pattern to Studio\# 1 in terms of the improvements achieved under the proposed interventions A1 (fig. 8A) and A2 (fig. 8B). In its current condition, Studio\#2 produces T30 results ranging from 1.34 to $2.11 \mathrm{~s}$ across the tested frequency range. In Proposal A2, the lowest T30 value is $1.1 \mathrm{~s}$, while in Proposal A1, the lowest value is $1.28 \mathrm{~s}$. At $125 \mathrm{~Hz}$, a reduction of $0.58 \mathrm{~s}$ is obtained in Proposal A2 (fig. 12B). The reason for this is that the sound absorption of the DNA5 configuration used in the proposed partition is high in this octave band.

The distribution maps in figure 13 show the STI results for the studios in their current condition and after the proposed AMM intervention (A2). In the scenario represented for Studio\#1, an instructor is talking to the whole class from a central position. While intelligibility in Studio\# 1 is generally "poor" in the current condition of the studio (fig. 13A), this improves to "fair" and "good" with the proposed installation of the AMM partitions (fig. 13B). For Studio\#2, the instructor is positioned to one side of the studio, which is a common situation when the studio is shared by two sections (classes) and two instructors. In the current condition of the studio, the STI values indicate "fair" intelligibility in most of the receiver positions on the same side of the partition where the instructor is standing (fig. 13C). However, after the proposed AMM intervention, the STI values show that "good" intelligibility is achieved at the drawing tables on the same side of the partition as the instructor (fig. 13D). These results highlight the improvements inside the studios and the potential benefits of the composite use of barrierplus-absorptive AMMs in a real architectural acoustics problem. Moreover, the proposed AMM applications do

FIG. 13 Studio\#1 STI distribution maps (A) for the current condition of the studio, $(B)$ under the A2 AMM proposal and Studio\#2 STI distribution maps, (C) for the current condition of the studio, and $(D)$ under the A2 AMM proposal.

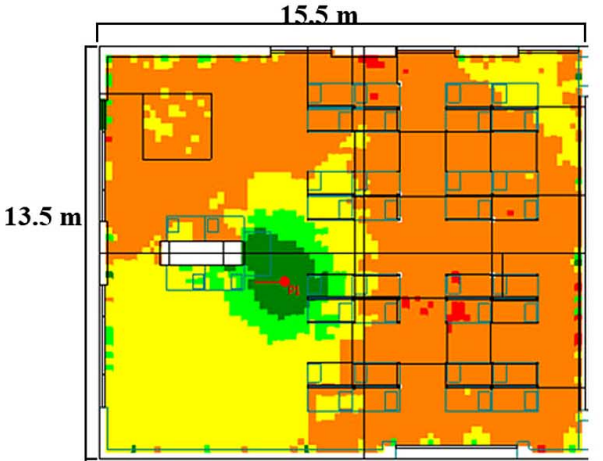

$(A)$

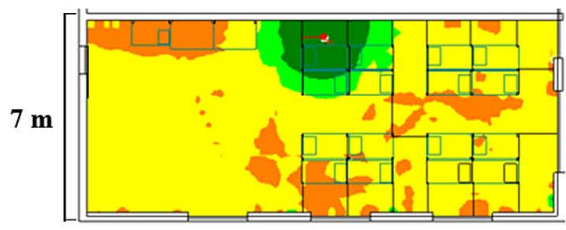

$(C)$

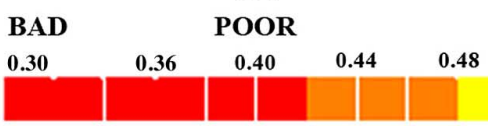

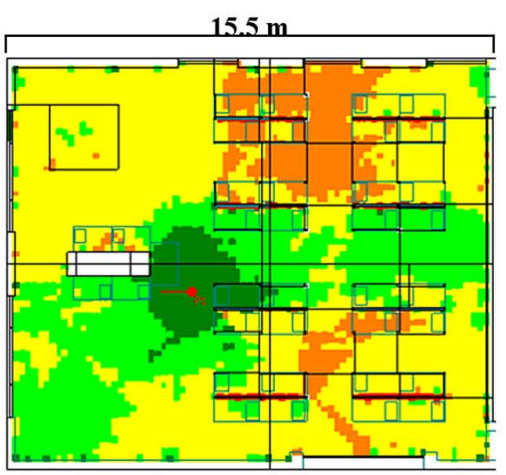

(B)

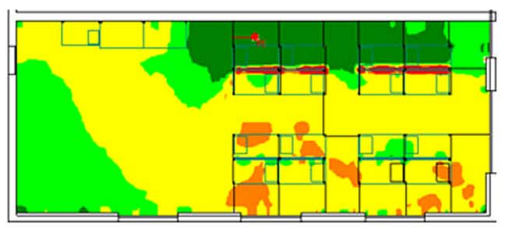

$(D)$

FAIR GOOD

$\begin{array}{lllll}0.52 & 0.56 & 0.60 & 0.64 & 0.70\end{array}$ 
not use porous materials, and it is always an option to improve the aesthetic features of the space in question through the use of integrated lighting over these semitranslucent prototypes.

\section{Conclusion}

In this study, different types of acoustical metamaterials have been developed to investigate their potential applications in architecture. The space-coiled modules that were developed utilize some forms and ratios taken from nature in line with a biomimicry approach, albeit on a subwavelength scale. Basically, three types of units named after the GR, the WL form, and DNA are designed and produced. The samples are then tested in an impedance tube to obtain their sound absorption coefficients and TL values.

The study focuses on acoustical enhancement in the midfrequency to low-frequency spectrum. This is because higher frequencies can be controlled easily with traditional materials without taking up much space inside the rooms. In addition, the presence of audiences or students augments the attenuation of sound in the midfrequency to high-frequency range. The analysis and the impedance tube tests are therefore conducted for frequencies between 63 and $1,000 \mathrm{~Hz}$ for sound absorption and frequencies between 100 and 1,000 $\mathrm{Hz}$ for sound TL. Different configurations of the proposed units are tested, including configurations with one side closed, with both sides open, with different thicknesses, and with different air gaps behind them. The results indicate that all the proposed AMM modules achieve higher sound absorption than a solid sample of the same thickness. The sample DNA module achieves the best overall sound absorption performance in the tested frequency ranges. Among the different configurations of this module, the DNA5 configuration $(8 \mathrm{~cm}$ thick, two sides open, $7.5 \mathrm{~cm}$ air gap behind) reaches the maximum sound absorption value of 0.77 at $63 \mathrm{~Hz}$. The basic drawback of the AMMs in sound absorption is their relatively narrow band behavior. Modules of two or three kinds, with alternating interior void dimensions, could be used jointly to increase the effective frequency interval.

The sample WL module achieved the highest performance in terms of sound TL, with values ranging from 48 to $55 \mathrm{~dB}$ across the entire tested frequency spectrum. The results achieved by the WL module are approximately $12 \mathrm{~dB}$ better than those obtained for a solid sample of the same thickness $(8 \mathrm{~cm})$. This is a significant contribution considering the relatively low thickness of the sample modules.

The sound TL achieved by the WL module is also compared with the sound TL achieved by panel walls made of the same base material (resin) in different configurations similar to those used in standard lightweight construction systems. For this purpose, TL results are obtained for theoretical panel wall sections consisting of two layers of solid resin panels of different thicknesses and different air gaps in between and with the same total thickness as the WL module. When the results are compared, the WL unit is found to display a greater TL performance than all of these estimated resin wall sections. The WL unit also performed better than a similar panel wall section with a mineral infill up to a frequency of $500 \mathrm{~Hz}$.

The impedance tube test results are also compared with two analytical methods, including the Helmholtz equation and PDI method. The analytical comparisons indicate that the DNA module cannot be explained by either the Helmholtz or PDI methods because of its complex inner structure. For this reason, the optimization studies of the DNA module for sound absorption should be followed by numerical simulations, which is one of the future steps of this research. On the other hand, GR and WL modules can partially be explained by PDI method for specifying their possible peak absorption frequencies during the design stage.

Finally, to observe the potential benefits in an architectural environment, partitions made up of AMMs are designed using the DNA5 module on one side and WL blocks on the other. This design solution is tested in two architectural studios by means of simulations over field test-tuned acoustical models. A comparison is made of the reverberation time (T30) and STI metrics for the studios in their current condition and under acoustical design scenarios using AMM partitions of different heights. The use of the partitions lowers the T30 values for Studio\#1 by $0.73 \mathrm{~s}$ and Studio\#2 by $0.69 \mathrm{~s}$ at $63 \mathrm{~Hz}$. The use of the partitions is also found to raise the STI values from "poor" to "fair" and "good" rating in Studio\#1 and from "fair" to "good" rating in Studio\#2. 
To sum up, the results highlight the potential use of AMMs in reverberation and noise control in architectural applications. AMMs can be utilized as part of a partition, as furniture, or as a design element without occupying large spaces and without using allergenic porous materials. For a similar reason of indoor air quality, AMMs can also be preferred as a diffuser in heating, ventilation, and air conditioning systems. That is, they can be utilized for attenuating noise without interrupting air flow and can support natural ventilation. On the other hand, as different manufacturing techniques make it possible to use a variety of base materials, including semitransparent and translucent materials, light can always be integrated into AMM modules as a design element. In acoustical terms, the inner geometrical structures of the modules proposed in this study can still be improved further to obtain higher values, either in absorption or TL, in a broader range of frequencies. In future work, the experimental results presented in this study will be utilized to simulate material properties for the optimization of the modules by a numerical implementation.

\section{ACKNOWLEDGMENTS}

Special thanks are extended to Renan Gökyay, partner of Nurus, for making the 3-D CNC printer available and for his special role in initiating this study on AMMs. The authors also acknowledge their gratitude to Professor Mehmet Çalışkan for his valuable consultations and to Mezzo Stüdyo for providing the laboratory facilities for the impedance tube measurements.

\section{References}

1. S. A. Cummer, J. Christensen, and A. Alù, “Controlling Sound with Acoustic Metamaterials," Nature Reviews Materials 1, no. 3 (March 2016): 16001, https://doi.org/10.1038/natrevmats.2016.1

2. Y. Chen, G. Hu, and G. Huang, "A Hybrid Elastic Metamaterial with Negative Mass Density and Tunable Bending Stiffness," Journal of the Mechanics and Physics of Solids 105 (August 2017): 179-198, https://doi.org/10.1016/j.jmps. 2017.05.009

3. M. I. Hussein, M. J. Leamy, and M. Ruzzene, "Dynamics of Phononic Materials and Structures: Historical Origins, Recent Progress, and Future Outlook," Applied Mechanics Reviews 66, no. 4 (July 2014): 040802, https://doi.org/10.1115/1. 4026911

4. E. Yablonovitch, "Inhibited Spontaneous Emission in Solid-State Physics and Electronics," Physical Review Letters 58, no. 20 (1987): 2059-2062, https://doi.org/10.1103/PhysRevLett.58.2059

5. F. dell'Isola, P. Seppecher, J. J. Alibert, T. Lekszycki, R. Grygoruk, M. Pawlikowski, D. Steigmann, et al., "Pantographic Metamaterials: An Example of Mathematically Driven Design and of Its Technological Challenges," Continuum Mechanics and Thermodynamics 31, no. 4 (2019): 851-884, https://doi.org/10.1007/s00161-018-0689-8

6. F. dell'Isola, P. Seppecher, M. Spagnuolo, E. Barchiesi, F. Hild, T. Lekszycki, I. Giorgio, et al., “Advances in Pantographic Structures: Design, Manufacturing, Models, Experiments and Image Analyses," Continuum Mechanics and Thermodynamics 31, no. 4 (2019): 1231-1282, https://doi.org/10.1007/s00161-019-00806-x

7. L. Zigoneanu, B.-I. Popa, and S. A. Cummer, "Three-Dimensional Broadband Omnidirectional Acoustic Ground Cloak," Nature Materials 13, no. 4 (April 2014): 352-355, https://doi.org/10.1038/nmat3901

8. L.-W. Cai and J. Sánchez-Dehesa, "Analysis of Cummer-Schurig Acoustic Cloaking," New Journal of Physics 9 , no. 12 (December 2007): 450, https://doi.org/10.1088/1367-2630/9/12/450

9. H. Ma, S. Qu, Z. Xu, and J. Wang, "Numerical Method for Designing Approximate Cloaks with Arbitrary Shapes," Physical Review E 78, no. 3 (September 2008): 036608, https://doi.org/10.1103/PhysRevE.78.036608

10. S. Zhang, C. Xia, and N. Fang, "Broadband Acoustic Cloak for Ultrasound Waves," Physical Review Letters 106, no. 2 (January 2011): 024301, https://doi.org/10.1103/PhysRevLett.106.024301

11. N. Kaina, F. Lemoult, M. Fink, and G. Lerosey, "Negative Refractive Index and Acoustic Superlens from Multiple Scattering in Single Negative Metamaterials," Nature 525, no. 7567 (September 2015): 77-81, https://doi.org/10.1038/ nature 14678

12. Y. Li, B. Liang, X. Tao, X. Zhu, X. Zou, and J. Cheng, “Acoustic Focusing by Coiling Up Space," Applied Physics Letters 101, no. 23 (December 2012): 233508, https://doi.org/10.1063/1.4769984

13. Y. Xie, W. Wang, H. Chen, A. Konneker, B.-I. Popa, and S. A. Cummer, "Wavefront Modulation and Subwavelength Diffractive Acoustics with an Acoustic Metasurface," Nature Communications 5, no. 1 (2014): 5553, https://doi.org/10. 1038/ncomms6553

14. V. M. García-Chocano, J. Christensen, and J. Sánchez-Dehesa, "Negative Refraction and Energy Funneling by Hyperbolic Materials: An Experimental Demonstration in Acoustics," Physical Review Letters 112, no. 14 (April 2014): 144301, https://doi.org/10.1103/PhysRevLett.112.144301 
15. Z. Liang and J. Li, "Extreme Acoustic Metamaterial by Coiling Up Space," Physical Review Letters 108, no. 11 (March 2012): 114301, https://doi.org/10.1103/PhysRevLett.108.114301

16. Y. Xie, B.-I. Popa, L. Zigoneanu, and S. A. Cummer, "Measurement of a Broadband Negative Index with Space-Coiling Acoustic Metamaterials," Physical Review Letters 110, no. 17 (April 2013): 175501, https://doi.org/10.1103/PhysRevLett. 110.175501

17. A. O. Krushynska, F. Bosia, M. Miniaci, and N. M. Pugno, "Fractal and Spider Web-Inspired Labyrinthine Acoustic Metamaterials," in 2017 11th International Congress on Engineered Materials Platforms for Novel Wave Phenomena (Metamaterials) (Piscataway, NJ: Institute of Electrical and Electronic Engineers, 2017), 187-189, http://doi.org/10. 1109/MetaMaterials.2017.8107885

18. C. R. Liu, J. H. Wu, K. Lu, Z. T. Zhao, and Z. Huang, “Acoustical Siphon Effect for Reducing the Thickness in MembraneType Metamaterials with Low-Frequency Broadband Absorption,” Applied Acoustics 148 (May 2019): 1-8, https://doi. org/10.1016/j.apacoust.2018.12.008

19. R. Fleury and A. Alù, "Extraordinary Sound Transmission through Density-Near-Zero Ultranarrow Channels," Physical Review Letters 111, no. 5 (July 2013): 055501, https://doi.org/10.1103/PhysRevLett.111.055501

20. C. Shen, J. Xu, N. X. Fang, and Y. Jing, "Anisotropic Complementary Acoustic Metamaterial for Canceling Out Aberrating Layers,” Physical Review X 4, no. 4 (2014): 041033, https://doi.org/10.1103/PhysRevX.4.041033

21. Z. Liu, X. Zhang, Y. Mao, Y. Y. Zhu, Z. Yang, C. T. Chan, and P. Sheng, "Locally Resonant Sonic Materials," Science 289, no. 5485 (September 2000): 1734-1736, https://doi.org/10.1126/science.289.5485.1734

22. N. Fang, D. Xi, J. Xu, M. Ambati, W. Srituravanich, C. Sun, and X. Zhang, "Ultrasonic Metamaterials with Negative Modulus," Nature Materials 5, no. 6 (June 2006): 452-456, https://doi.org/10.1038/nmat1644

23. B.-I. Popa, L. Zigoneanu, and S. A. Cummer, "Experimental Acoustic Ground Cloak in Air," Physical Review Letters 106, no. 25 (June 2011): 253901, http://doi.org/10.1103/PhysRevLett.106.253901

24. Z. Liang, T. Feng, S. Lok, F. Liu, K. B. Ng, C. H. Chan, J. Wang, et al., "Space-Coiling Metamaterials with Double Negativity and Conical Dispersion," Scientific Reports 3, no. 1 (December 2013): 1614, https://doi.org/10.1038/srep01614

25. X. Wang, Y. Zhou, J. Sang, and W. Zhu, “A Generalized Model for Space-Coiling Resonators," Applied Acoustics 158 (January 2020): 107045, https://doi.org/10.1016/j.apacoust.2019.107045

26. Y. Cheng, C. Zhou, B. G. Yuan, D. J. Wu, Q. Wei, and X. J. Liu, "Ultra-Sparse Metasurface for High Reflection of LowFrequency Sound Based on Artificial Mie Resonances," Nature Materials 14, no. 10 (October 2015): 1013-1019, https:// doi.org/10.1038/nmat4393

27. Z. Yang, H. M. Dai, N. H. Chan, G. C. Ma, and P. Sheng, "Acoustic Metamaterial Panels for Sound Attenuation in the 501000 Hz Regime,” Applied Physics Letters 96, no. 4 (January 2010): 041906, https://doi.org/10.1063/1.3299007

28. T. Huang, C. Shen, and Y. Jing, "Membrane- and Plate-Type Acoustic Metamaterials," The Journal of the Acoustical Society of America 139, no. 6 (June 2016): 3240-3250, https://doi.org/10.1121/1.4950751

29. T. Frenzel, J. D. Brehm, T. Bückmann, R. Schittny, M. Kadic, and M. Wegener, "Three-Dimensional Labyrinthine Acoustic Metamaterials," Applied Physics Letters 103, no. 6 (August 2013): 061907, https://doi.org/10.1063/1.4817934

30. L. Yong, B. Liang, Z. Gu, X. Zou, and J. Cheng, "Reflected Wavefront Manipulation Based on Ultrathin Planar Acoustic Metasurfaces," Scientific Reports 3, no. 1 (2013): 2546, https://doi.org/10.1038/srep02546

31. C. Liu, B. Xia, and D. Yu, "The Spiral-Labyrinthine Acoustic Metamaterial by Coiling Up Space," Physics Letters A 381, no. 36 (September 2017): 3112-3118, https://doi.org/10.1016/j.physleta.2017.07.041

32. O. B. Godbold, R. C. Soar, and R. A. Buswell, "Implications of Solid Freeform Fabrication on Acoustic Absorbers," Rapid Prototyping Journal 13, no. 5 (October 2007): 298-303, https://doi.org/10.1108/13552540710824805

33. F. Setaki, M. Tenpierik, M. Turrin, and A. van Timmeren, "Acoustic Absorbers by Additive Manufacturing," Building and Environment 72 (February 2014): 188-200, https://doi.org/10.1016/j.buildenv.2013.10.010

34. Y. Bar-Cohen, Biomimetics: Biologically Inspired Technologies (Boca Raton, FL: CRC Press, 2006).

35. M. Akhtaruzzaman and A. A. Shafie, "Geometrical Substantiation of Phi, the Golden Ratio and the Baroque of Nature, Architecture, Design and Engineering,” International Journal of Arts 1, no. 1 (2011): 1-22, http://doi.org/10.5923/j.arts. 20110101.01

36. N. Sui, X. Yan, T.-Y. Huang, J. Xu, F.-G. Yuan, and Y. Jing, “A Lightweight Yet Sound-Proof Honeycomb Acoustic Metamaterial," Applied Physics Letters 106, no. 17 (April 2015): 171905, https://doi.org/10.1063/1.4919235

37. C. J. Alden and S.-H. Kim, "Solvent-Accessible Surfaces of Nucleic Acids," Journal of Molecular Biology 132, no. 3 (August 1979): 411-434, https://doi.org/10.1016/0022-2836(79)90268-7

38. A. D. Smith and J. P. Bolam, "The Neural Network of the Basal Ganglia as Revealed by the Study of Synaptic Connections of Identified Neurones," Trends in Neurosciences 13, no. 7 (July 1990): 259-265, https://doi.org/10.1016/0166-2236(90) 90106-K

39. D. C. Phillips, “The Three-Dimensional Structure of an Enzyme Molecule," Scientific American 215, no. 5 (November 1966): 78-90, http://web.archive.org/web/20201208205920/https://www.jstor.org/stable/24931327

40. M. Vomhof, L. Vasey, F. Gramazio, M. Kohler, S. Bräuer, K. Eggenschwiler, and J. Strauss, "Robotic Fabrication of Acoustic Brick Walls," in ACADIA 2014 Design Agency: Proceedings of the 34th Annual Conference of the Association for Computer Aided Design in Architecture, ed. D. Gerber, A. Huang, and J. Sanchez (Ontario, Canada: Riverside Architectural Press, 2014), 555-564.

41. K.-M. M. Tam and C. T. Mueller, “Additive Manufacturing Along Principal Stress Lines," 3D Printing and Additive Manufacturing 4, no. 2 (June 2017): 63-81, https://doi.org/10.1089/3dp.2017.0001 
42. S. Lim, R. A. Buswell, T. T. Le, S. A. Austin, A. G. F. Gibb, and T. Thorpe, "Developments in Construction-Scale Additive Manufacturing Processes," Automation in Construction 21 (January 2012): 262-268, https://doi.org/10.1016/j.autcon. 2011.06.010

43. Z. Quan, A. Wu, M. Keefe, X. Qin, J. Yu, J. Suhr, J.-H. Byun, B.-S. Kim, and T.-W. Chou, "Additive Manufacturing of Multi-Directional Preforms for Composites: Opportunities and Challenges," Materials Today 18, no. 9 (November 2015): 503-512, https://doi.org/10.1016/j.mattod.2015.05.001

44. Acoustics - Determination of Sound Absorption Coefficient and Impedance in Impedance Tubes - Part 2: TransferFunction Method, ISO (Geneva, Switzerland: International Organization for Standardization, 1998).

45. Y. Xishan, J. Yin, G. Yu, L. Peng, and N. Wang, “Acoustic Superlens Using Helmholtz-Resonator-Based Metamaterials," Applied Physics Letters 107, no. 19 (2015): 193505, https://doi.org/10.1063/1.4935589

46. T. Yamamoto, "Acoustic Metamaterial Plate Embedded with Helmholtz Resonators for Extraordinary Sound Transmission Loss," Journal of Applied Physics 123, no. 21 (2018): 215110, https://doi.org/10.1063/1.5025570

47. M. Long, Architectural Acoustics (Oxford, UK: Academic Press, 2014).

48. T. Lato and A. Mohany, "Passive Damping of Pressure Pulsations in Pipelines Using Herschel-Quincke Tubes," Journal of Sound and Vibration 448 (May 2019): 160-177, https://doi.org/10.1016/j.jsv.2019.02.020

49. M. R. Haberman and M. D. Guild, “Acoustic Metamaterials,” Physics Today 69, no. 6 (June 2016): 42-48, https://doi.org/ 10.1063/PT.3.3198

50. S. Varanasi, J. S. Bolton, and T. Siegmund, "Experiments on the Low Frequency Barrier Characteristics of Cellular Metamaterial Panels in a Diffuse Sound Field," The Journal of the Acoustical Society of America 141, no. 1 (January 2017): 602-610, https://doi.org/10.1121/1.4974257

51. S. Babaee, J. T. B. Overvelde, E. R. Chen, V. Tournat, and K. Bertoldi, "Reconfigurable Origami-Inspired Acoustic Waveguides," Science Advances 2, no. 11 (November 2016): e1601019, https://doi.org/10.1126/sciadv.1601019 